\title{
A NO'TE ON EULER NUMBERS AND POLYNOMIALS
}

\author{
L. CARLITZ
}

1. Euler numbers. Let $E_{m}$ denote the Euler number in the even suffix notation so that

$$
(E+1)^{m}+(E-1)^{m}=0 \quad(m>0), \quad E_{0}=1,
$$

where, as usual, after expansion of the left member $E^{r}$ is replaced by $E_{r}$. Nielsen [4, p. 273] has proved that

$$
E_{2 m} \equiv\left\{\begin{array}{llll}
0 & (\bmod p) & (p \equiv 1 & (\bmod 4)) \\
2 & (\bmod p) & (p \equiv 3 & (\bmod 4)),
\end{array}\right.
$$

where $p$ is an odd prime such that $p-1 \mid 2 m$. The special case $m=p-1$ is due to Ely $[1, p, 341]$.

We wish to point out, to begin with, that (1.2) can be extended to give

$$
E_{2 m} \equiv\left\{\begin{array}{llll}
0 & \left(\bmod p^{e}\right) & (p \equiv 1 & (\bmod 4)) \\
2 & \left(\bmod p^{e}\right) & (p \equiv 3 & (\bmod 4))
\end{array}\right.
$$

where $p$ is an odd prime such that $(p-1) p^{e-1} \mid 2 m$.

To prove (1.3) we begin with the formula

$$
E_{m}(x+1)+E_{m}(x)=2 x^{m},
$$

where $[5$, p. 25$]$

$$
E_{m}(x)=\sum_{0 \leqq 2 s \leqq m}\left(\begin{array}{c}
m \\
2 s
\end{array}\right) 2^{-2 s}\left(x-\frac{1}{2}\right)^{m-2 s} E_{2 s}
$$

is the Euler polynomial of degree $m$. It is clear from (1.4) that

$$
2 \sum_{s=0}^{r}(-1)^{s}(x+s)^{m}=E_{m}(x)+(-1)^{r} E_{m}(x+r+1) .
$$

We also recall that $[5$, p. 28$]$

Received Feb. 18, 1954. 


$$
E_{m}(x)=\sum_{s=0}^{m}\left(\begin{array}{c}
m \\
s
\end{array}\right) 2^{-s} C_{s} x^{m-s}
$$

where

$$
C_{m-1}=2^{m}\left(1-2^{m}\right) \frac{B_{m}}{m} ; \quad C_{0}=1, \quad C_{2 r}=0 \quad(r \geqslant 1) .
$$

Consequently for $x=0,(1.5)$ and (1.6) imply

$$
2 \sum_{s=1}^{r}(-1)^{r-s} s^{2 m}=E_{2 m}(r+1)=2^{-2 m} \sum_{s=0}^{m}\left(\begin{array}{c}
2 m \\
2 s
\end{array}\right)(2 r+1)^{2 m-2 s} E_{2 s} .
$$

Clearly (1.9) yields the congruence

$$
2^{2 m+1} \sum_{s=1}^{r}(-1)^{r-s} s^{2 m} \equiv E_{2 m} \quad\left(\bmod (2 r+1)^{2}\right) .
$$

Now let $(p-1) p^{e-1} \mid 2 m$ and $p^{e} \mid(2 r+1)^{2}$. Then for $p+s$ it is evident that $s^{2 m} \equiv 1\left(\bmod p^{e}\right)$, while for $p \mid s$ we have $s^{2 m} \equiv 0\left(\bmod p^{e}\right)$. Thus the left member of (1.10) is congruent to

$$
2 \sum_{\substack{s=1 \\ p+s}}^{r}(-1)^{r-s} \quad\left(\bmod p^{e}\right)
$$

Since $p \mid 2 r+1$ implies $r \equiv \frac{1}{2}(p-1)(\bmod p)$, it follows at once that $(1.11)$ reduces to

$$
2\left(1-1+\ldots+(-1)^{\frac{1}{2}(p-3)}\right)=\left\{\begin{array}{lll}
0 & (p \equiv 1 & (\bmod 4)) \\
2 & (p \equiv 3 & (\bmod 4))
\end{array}\right.
$$

Comparison of (1.10) and (1.12) leads at once to (1.3). This proves

TheOREM 1. If $(p-1) p^{e-1} \mid 2 m$ then (1.3) holds.

For a different proof of (1.3) see [2, p. 845].

2. Euler polynomials. Returning to (1.6) we put $x=a$, where $a$ is a rational umber that is integral $(\bmod p)$. Since for $a \equiv b\left(\bmod p^{e}\right)$ we have $E_{m}(a) \equiv E_{m}(b)\left(\bmod p^{e}\right)$, there is no loss in generality in assuming that $a$ is an integer.

If we take $r=p-1,(1.6)$ becomes

$$
2 \sum_{s=0}^{p-1}(-1)^{s}(a+s)^{2 m}=E_{2 m}(a)+E_{2 m}(a+p) .
$$


Let $a \equiv 0(\bmod p)$ and assume that $(p-1) p^{e-1} \mid 2 m$. Then $(2.1)$ reduces to

$$
E_{2 m}(a)+E_{2 m}(a+p) \equiv 0 \quad\left(\bmod p^{e}\right) .
$$

Since by (1.7) and (1.8), $E_{2 m}(0)=0$ for $m \geqslant 1$ we therefore get from (2.2)

$$
E_{2 m}(a) \equiv 0 \quad\left(\bmod p^{e}\right) \quad(p \mid a) .
$$

For $a \equiv 1(\bmod p)$ it is also clear that the left member of $(2.1)$ is divisible by $p^{e}$; since $E_{2 m}(1)=0$ for $m \geq 1$ we get

$$
E_{2 m}(a) \equiv 0 \quad\left(\bmod p^{e}\right) \quad(a \equiv 1 \quad(\bmod p)) .
$$

In the next place, since

$$
E_{m}(x+r)=\sum_{s=0}^{m}\left(\begin{array}{c}
m \\
s
\end{array}\right) r^{m-s} E_{s}(x),
$$

it follows from (1.6) that

$$
\begin{aligned}
2 \sum_{s=0}^{r-1}( & -1)^{s}(a+s)^{2 m} \\
& =\left(1+(-1)^{r-1}\right) E_{2 m}(a)+(-1)^{r-1} \sum_{s=0}^{2 m-1}\left(\begin{array}{c}
2 m \\
s
\end{array}\right) r^{2 m-s} E_{s}(a) \\
& \equiv\left(1+(-1)^{r-1}\right) E_{2 m}(a) \quad(\bmod r) .
\end{aligned}
$$

We take $r$ odd, $p^{e} \mid r$ and $(p-1) p^{e-1} \mid 2 m$; since

$$
(a+p)^{2 m} \equiv a^{2 m} \quad\left(\bmod p^{e}\right),
$$

it follows at once from (2.5) that

$$
E_{2 m}(a+p) \equiv E_{2 m}(a) \quad\left(\bmod p^{e}\right),
$$

where $a$ is arbitrary (but integral $(\bmod p)$ ).

Thus to determine the residue of $E_{2 m}(a)$ it suffices to take $1 \leqq a \leqq p-1$. Using (1.6) we have

$$
2 \sum_{s=0}^{r}(-1)^{r-s}(a+s)^{2 m}=(-1)^{r} E_{2 m}(a)+E_{2 m}(a+r+1),
$$

which implies

$$
\begin{aligned}
2 \sum_{s=0}^{r}(-1)^{r-s}(a+s)^{2 m} \equiv(-1)^{r} E_{2 m}(a) & +2^{-2 m} E_{2 m} \\
& \left(\bmod (2 a+2 r+1)^{2}\right) .
\end{aligned}
$$

If we assume that $(p-1) p^{e-1} \mid 2 m$ and $p^{e} \mid(2 a+2 r+1)^{2}$ then (2.7) becomes 


$$
2 \sum_{\substack{s=0 \\ p+a+s}}^{r}(-1)^{r-s} \equiv(-1)^{r} E_{2 m}(a)+E_{2 m} \quad\left(\bmod p^{e}\right) .
$$

Clearly the left member of (2.8) is equal to

$$
2 \sum_{\substack{s=1 \\ p+s}}^{a+r}(-1)^{a+r-s}-2 \sum_{s=1}^{a-1}(-1)^{a+r-s}
$$

Comparing the first sum in (2.9) with (1.11) and using (1.3) it is clear that (2.8) becomes

$$
(-1)^{r} E_{2 m}(a) \equiv-2 \sum_{s=1}^{a-1}(-1)^{a+r-s}
$$

and therefore finally

$$
E_{2 m}(a) \equiv 1+(-1)^{a} \quad\left(\bmod p^{e}\right) \quad(1 \leqq a \leqq p-1) .
$$

We may state

THEOREM 2. If $(p-1) p^{e-1} \mid 2 m$ and $p+a$ then

$$
E_{2 m}(a) \equiv 1+(-1)^{c} \quad\left(\bmod p^{e}\right),
$$

where $a \equiv c(\bmod p), 1 \leqq c \leqq p-1$; if $p \mid a$, then (2.3) holds.

It is evident that (2.11) includes (2.4); also it is not difficult to show that (2.11) includes (1.3).

3. Additional results. If in (1.6) we replace $m$ by $2 m-1$ we get using (1.5)

$$
2 \sum_{s=0}^{r}(-1)^{s}(a+s)^{2 m-1} \equiv E_{2 m-1}(a) \quad(\bmod 2 a+2 r+1) .
$$

Hence if $(p-1) p^{e-1} \mid 2 m$ and $p^{e} \mid 2 a+2 r+1$, (3.1) implies

$$
2 \sum_{\substack{s=0 \\ p+a+s}}^{r} \frac{(-1)^{s}}{a+s} \equiv E_{2 m-1}(a) \quad\left(\bmod p^{e}\right)
$$

In particular when $a=0$, it follows from (1.8) that

$$
\sum_{\substack{s=0 \\ p+s}}^{\frac{1}{2}\left(p^{e}-1\right)} \frac{(-1)^{s}}{s} \equiv C_{2 m-1} \equiv\left(1-2^{2 m}\right) \frac{B_{2 m}}{2 m} \quad\left(\bmod p^{e}\right) ;
$$

the special case 


$$
\sum_{s=0}^{3(p-1)} \frac{(-1)^{s}}{s} \equiv C_{2 m-1} \quad(\bmod p) \quad(p-1 \mid 2 m)
$$

may be noted. We also remark that for $a=\frac{1}{2},(3.2)$ becomes

$$
\sum_{\substack{s=0 \\ p+2 s+1}}^{p^{e}} \frac{(-1)^{s}}{2 s+1} \equiv 0 \quad\left(\bmod p^{e}\right) .
$$

For formulas like (3.4) see Glaisher [3].

If $(a / p)$ denotes the Legendre symbol, then

$$
a^{2^{1}(p-1) p^{e-1}} \equiv\left(\frac{a}{p}\right) \quad\left(\bmod p^{e}\right)
$$

Thus (1.6) implies

$$
2 \sum_{s=0}^{r-1}(-1)^{s}\left(\frac{a+s}{p}\right) \equiv E_{m}(a)+(-1)^{r-1} E_{m}(a+r) \quad\left(\bmod p^{e}\right),
$$

where $m$ is an odd multiple of $\frac{1}{2}(p-1) p^{e-1}$. Now let $r$ be odd, $p^{e} \mid r$; then (3.6) yields

$$
\sum_{s=0}^{r-1}(-1)^{s}\left(\frac{a+s}{p}\right) \equiv E_{m}(a) \quad\left(\bmod p^{e}\right)
$$

It follows at once from (3.7) that

$$
E_{m}(a+p) \equiv E_{m}(a) \quad\left(\bmod p^{e}\right) .
$$

Moreover it is clear from (3.7) that $(r=p t)$

$$
\begin{aligned}
E_{m}(a) & \equiv \sum_{j=0}^{t-1} \sum_{i=0}^{p-1}(-1)^{i+p j}\left(\frac{a+i}{p}\right) \\
& \equiv \sum_{j=0}^{t-1}(-1)^{j} \sum_{i=0}^{p-1}(-1)^{i}\left(\frac{a+i}{p}\right) \equiv \sum_{i=0}^{p-1}(-1)^{i}\left(\frac{a+i}{p}\right),
\end{aligned}
$$

so that

$$
E_{m}(a) \equiv \sum_{i=0}^{p-1}(-1)^{i}\left(\frac{a+i}{p}\right) \quad\left(\bmod p^{e}\right)
$$

In particular for $a=0,(3.9)$ becomes

$$
E_{m}(0) \equiv \sum_{i=0}^{p-1}(-1)^{i}\left(\frac{i}{p}\right) \quad\left(\bmod p^{e}\right) .
$$

For $p \equiv 1(\bmod 4)$, both members of $(3.10)$ vanish, while for $p \equiv 3(\bmod 4)$ 
we get

$$
\boldsymbol{C}_{m} \equiv 2 \sum_{s=0}^{\frac{1}{2}(p-1)}(-1)^{s}\left(\frac{2 s}{p}\right) \quad\left(\bmod p^{e}\right)
$$

Let $1 \leqq a \leqq p-1$; then by (3.9)

$$
\begin{aligned}
E_{m}(a) & \equiv(-1)^{a} \sum_{s=a}^{p+a-1}(-1)^{s}\left(\frac{s}{p}\right) \\
& \equiv(-1)^{a} \sum_{s=0}^{p-1}(-1)^{s}\left(\frac{s}{p}\right)-2(-1)^{a} \sum_{s=0}^{a-1}(-1)^{s}\left(\frac{s}{p}\right) .
\end{aligned}
$$

Comparing with (3.10) we get

$$
E_{m}(0)-E_{m}(a) \equiv 2(-1)^{a} \sum_{s=0}^{a-1}(-1)^{s}\left(\frac{s}{p}\right) \quad\left(\bmod p^{e}\right) .
$$

We may state

TheOREM 3. If $m$ is an odd multiple of $\frac{1}{2}(p-1) p^{e-1}$, then (3.8), (3.10) and (3.12) hold.

In particular, (3.12) implies

$$
C_{m}-E_{m} \equiv 2(-1)^{\frac{1}{2}(p+1)} \sum_{s=0}^{\frac{1}{2}(p-1)}(-1)^{s}\left(\frac{2 s}{p}\right) \quad\left(\bmod p^{e}\right),
$$

which includes (3.11).

4. Eulerian numbers and polynomials. It is of interest to compare (2.3) with the following known results for Bernoulli polynomials.

$$
\begin{gathered}
B_{m}(a) \equiv 0 \quad\left(\bmod p^{e}\right) \quad\left(p^{e} \mid m, p-1+m\right), \\
B_{m}(a)+\frac{1}{p}-1 \equiv 0 \quad\left(\bmod p^{e}\right) \quad\left((p-1) p^{e} \mid m\right),
\end{gathered}
$$

where the rational number $a$ is integral $(\bmod p)$. However it seems more instructive to discuss the "Eulerian" numbers $\phi_{m}(\zeta)$ defined by

$$
\frac{1-\zeta}{e^{t}-\zeta}=\sum_{m=0}^{\infty} \phi_{m}(\zeta) \frac{t^{m}}{m !} \quad(\zeta \neq 1),
$$

and the polynomials

$$
\phi_{m}(x, \zeta)=\sum_{s=0}^{m}\left(\begin{array}{c}
m \\
s
\end{array}\right) x^{m-s} \phi_{s}(\zeta)=(x+\phi(\zeta))^{m} .
$$

For a detailed study of $\phi_{m}(\zeta)$ see [2]. We shall suppose that the parameter $\zeta$ 
is an $l$-th root of unity, where $l \geqslant 2$.

It is an immediate consequence of (4.4) that

$$
\phi_{m}(x+1, \zeta)-\zeta \phi_{m}(x, \zeta)=(1-\zeta) x^{n} .
$$

(Since $\phi_{m}(x,-1)=E_{m}(x)$, it is clear that $(4.5)$ reduces to $(1.4)$ when $\zeta=-1$ ). By means of $(4.5)$ we readily obtain

$$
\phi_{m}(x+r, \zeta)-\zeta^{r} \phi_{m}(x, \zeta)=(1-\zeta) \sum_{s=0}^{r-1} \zeta^{r-1-s}(x+s)^{m}
$$

Substituting from (4.4) it is evident that (4.6) implies

$$
\begin{aligned}
\left(1-\zeta^{r}\right) \phi_{m}(x, \zeta)+\sum_{s=1}^{m-1}\left(\begin{array}{c}
m \\
s
\end{array}\right) r^{m-s} \phi_{m}(x, \zeta) & \\
& =(1-\zeta) \sum_{s=0}^{r-1} \zeta^{r-1-s}(x+s)^{m} .
\end{aligned}
$$

Now replace $x$ by a rational number $a$ that is integral $(\bmod p)$. The number $\phi_{m l}(\zeta)$ is in the field $R(\zeta)$, where $R$ is the rational field; more precisely it is of the form $\alpha_{m} /(1-\zeta)^{m}$, where $\alpha_{m}$ is an integer of $R(\zeta)$. If we assume that $(p, 1-\zeta)=(1)$, then $\phi_{m}(\zeta)$ is integral $(\bmod p)$; the same is therefore true of $\phi_{m}(a, \zeta)$. In the next place (4.7) implies

$$
\left(1-\zeta^{r}\right) \phi_{m}(x, \zeta) \equiv(1-\zeta) \sum_{s=0}^{r-1} \zeta^{r-1-s}(x+s)^{m} \quad(\bmod r),
$$

provided $(r, 1-\zeta)=(1)$. Let us now assume that $(p-1) p^{e-1} \mid m$ and $p^{e} \mid r$. Then $(4.8)$ reduces to

$$
\left(1-\zeta^{r}\right) \phi_{m}(a, \zeta) \equiv(1-\zeta) \sum_{\substack{s=0 \\ p+a+s}}^{r-1} \zeta^{r-1-s} \quad\left(\bmod p^{e}\right)
$$

If we suppose, as we may, that $l+r$, then it follows readily from (4.9) that

$$
\phi_{m}(a+p, \zeta) \equiv \phi_{m}(a, \zeta) \quad\left(\bmod p^{e}\right) .
$$

It accordingly suffices to assume that $0 \leqq a \leqq p-1$.

In the first place for $a=0,(4.9)$ reduces to

$$
\left(1-\zeta^{r}\right) \phi_{m}(\zeta) \equiv(1-\zeta) \sum_{\substack{s=0 \\ p+s}}^{r-1} \zeta^{r-1-s} \quad\left(\bmod p^{e}\right) .
$$

We shall take $r \equiv 1(\bmod l)$; then $(4.11)$ gives 


$$
\phi_{m}(\zeta) \equiv \sum_{s=0}^{r-1} \zeta^{r-1-s}-\sum_{s=0}^{t-1} \zeta^{r-1-p s}
$$

where $r=t p$. A little computation now gives

$$
\phi_{m}(\zeta)=\frac{1-\zeta^{p-1}}{1-\zeta^{p}} \quad\left(\bmod p^{e}\right)
$$

Next for $1 \leqq a \leqq p-1$, where again $r \equiv 1(\bmod l), r=t p$, it follows from (4.9) that

$$
\begin{aligned}
\phi_{m}(a, \zeta) & \equiv \sum_{s=0}^{a+r-1} \zeta^{a+r-1-s}-\sum_{s=0}^{a-1} \zeta^{a+r-1-s}-\sum_{s=1}^{t} \zeta^{a+r-1-p s} \\
& \equiv \frac{1-\zeta^{a+r}}{1-\zeta}-\zeta^{r} \frac{1-\zeta^{a}}{1-\zeta}-\zeta^{a-1} \frac{1-\zeta^{p t}}{1-\zeta^{p}} \\
& \equiv 1-\zeta^{a-1} \frac{1-\zeta}{1-\zeta^{p}} .
\end{aligned}
$$

Hence using (4.10) we get

$$
\phi_{m}(a, \zeta) \equiv 1-\zeta^{c-1} \frac{1-\zeta}{1-\zeta^{p}} \quad\left(\bmod p^{e}\right)
$$

where $a \equiv c(\bmod p), 1 \leqq c \leqq p-1$. This completes the proof of

THEOREM 4. Let $(p-1) p^{e-1} \mid m$ and let $a \equiv c(\bmod p)$, where $0 \leqq c \leqq p-1$. Then if $c \neq 0$, (4.13) holds, while for $c=0$ we have

$$
\phi_{m}(a, \zeta) \equiv \frac{1-\zeta^{p-1}}{1-\zeta^{p}}\left(\bmod p^{e}\right) \quad(p \mid a) .
$$

It is clear that for $\zeta=-1,(4.13)$ reduces to (2.11) and (4.14) reduces to (2.3). For the special case $a=0$ of (4.14) see [2, p. 842].

If $\alpha$ is an integer of $R(\zeta)$, we may again employ (4.8). Let $p$ be a prime ideal of $R(\zeta), N \mathfrak{p}=p^{f}$, where $(p, l)=1$. Then if we assume that

$$
(N \mathfrak{p}-1) p^{e-1} \mid m,
$$

and $p^{e} \mid r$, we get

$$
\left(1-\zeta^{r}\right) \phi_{m}(\alpha, \zeta) \equiv(1-\zeta) \sum_{\substack{s=0 \\ p+\alpha+s}}^{r-1} \zeta^{r-1-s} \quad\left(\bmod p^{e}\right)
$$

It follows that if $\pi \in \mathfrak{p}$ then

$$
\phi_{m}(\alpha+\pi, \zeta) \equiv \phi_{m}(\alpha, \zeta) \quad\left(\bmod p^{e}\right),
$$

and therefore 


$$
\phi_{m}(\alpha+p, \zeta) \equiv \phi_{m}(\alpha, \zeta) \quad\left(\bmod p^{e}\right) .
$$

Now if $u$ is congruent to a rational integer $(\bmod p)$, then, in view of (4.17), (4.13) holds. On the other hand, when $\alpha$ is not congruent to a rational integer, then in the right member of (4.16) the condition $\mathfrak{p}+\alpha+s$ is satisfied automatically and we get $(r \equiv 1(\bmod l))$

$$
\phi_{m}(a, \zeta) \equiv \sum_{s=0}^{r-1} \zeta^{s} \equiv \frac{1-\zeta^{r}}{1-\zeta} \equiv 1 \quad\left(\bmod p^{e}\right) .
$$

We may state

TheOREm 5. Let $\alpha$ be an integer of $R(\zeta), p+l$, and assume that (4.15) is satisfied, where $p$ is a prime ideal of $R(\zeta), N \mathfrak{p}=p^{f}$. Then if $\alpha$ is congruent to a rational integer $a(\bmod \mathfrak{p}),(4.13)$ and $(4.14)$ hold; otherwise we have

$$
\phi_{m}(\alpha, \zeta) \equiv 1 \quad\left(\bmod p^{e}\right) .
$$

In particular if $N p=p$, (4.13) and (4.14) apply.

\section{REFERENCES}

[1] G. S. Ely, Some notes on the numbers of Bernoulli and Euler, American Journal of Mathematics, Vol. 5(1880), pp. 337-341.

[2] G. Frobenius, Über die Bernoulli'schen Zahlen und die Euler'schen Polynome, Sitzungsberichte der Preussischen Akademie der Wissenschaften (1910), pp. 809-847.

[3] W. L. G. Glaisher, On the residues of the sums of the inverse powers of numbers in arithmetical progression, Quarterly Journal of Mathematics, Vol. 32 (1901), pp. 271-30).

[4] N. Nielsen, Traité élémentaire des nombres de Bernoulli, Paris, 1923.

[5] N. E. Nörlund, Vorlesungen über Differenzenrechnung, Berlin, 1924.

\section{Duke University}

